\title{
Analysis and Simulation of Auxiliary Space-based Measurement and Control Method Based on Beidou Navigation System
}

\author{
Shuaihe Gao ${ }^{\mathrm{a}}$, Lin Chen, Yi Zhou, Lishu Guo \\ The 29th Research Institute of China Electronics Technology Group Corporation \\ agaoshuaihe@aliyun.com
}

Keywords: Space-based measurement and control, Beidou navigation system, measuring radian, spread ability.

Abstract: The number of data relay satellites is limited, which will bring the spread ability to be a low level. To enhance the spread ability of tracking and measuring, the real-time performance of data transmission, and the reliability of information dissemination, the method of constructing data relay link by Beidou system is researched. Through design and simulation, the auxiliary means of space-based measurement and control by Beidou is validated.

\section{Introduction}

With the increasing in the space launch missions and the increasing in the requirements of measuring and controlling, the development of ground-based measurement and control systems, and sea-based measurement and control systems have been unable to fully meet the needs of rocket and satellite monitoring and control tasks. It is mainly manifested that blind areas for observation and control, lack of real-time in satellite payload data transmission, and difficult to achieve emergency monitoring and control of satellites and rockets.

In the 1980s, space-based measurement and control systems were gradually implemented. At present, the United States has completed the deployment of the second generation of Tacking and Data Relay Satellite (TDRS), and is developing third-generation TDRS. Russia's relay satellite system has provided monitoring and data transmission support for such missions as low-orbit satellites, space stations, alliance spacecraft. In Europe, the development of European Data Relay Satellite was carried out, and laser transmission experiment was carried out on 2 satellites. Japan expects to launch second-generation Data Relay and Tracking Satellite with inter-satellite optical communication links in 2019. The rate of optical link in the future DRTS is expected to exceed $10 \mathrm{G}$ bits /s. China is also continuously constructing and developing relay satellite systems, and has successfully applied in tasks such as the Shenzhou space flight and Tiangong mission.

Because of the limited number of data relay satellites, if the satellite is not available, the coverage 
of the system will be greatly reduced. At present, adding on-orbit satellites and improving the system's disaster recovery capability are considered to enhance the reliability and availability of the entire system. In order to greatly improve the coverage of measurement and control, the real-time nature of data transmission, and the reliability of measurement and control of data transmission, the feasibility of using Beidou constellation to construct space-based measurement and control means is researched. Through design and simulation, the auxiliary means of space-based measurement and control by Beidou is validated.

\section{System analysis}

The design idea of using the Beidou constellation to form space-based monitoring and control means is to directly communicate with its domestic stations when observation targets are visible, and to relay by the Beidou Global System for the observation and control tasks when the space-based targets are not visible to the domestic stations.

Inter-satellite links are existed in Beidou system, which can be used as relay forwarding channels for measurement and control tasks. Through reasonable design and planning, the Beidou Global System can be used for the real-time monitoring and control of space-based targets in the full airspace.

According to the above design of inter-satellite, the principle of data relaying path through Beidou Global System is that the transmission stable without interruption, the number of transit, the priority in the same layer, and the channel performance should be considered comprehensively. Therefore, it is recommended to choose the geosynchronous earth orbit (GEO) satellites firstly, and then inclined geosynchronous satellite orbit (IGSO) satellites and medium earth orbit (MEO) satellites.

\section{Simulation analysis}

Through the basic parameters (such as orientation, orbital radius, and etc.) of Beidou Global System, the models of satellites, ground station are established. Based on the models, link simulations are performed on the mission support schemes of the GEOs, IGSOs, and MEOs in the Beidou navigation system. Based on the link simulations, the monitoring and control coverage analysis of the GEO satellites, IGSO satellites and MEO satellites support schemes was performed respectively.

\subsection{Communication link simulation}

The BeiDou navigation system supports the entire airspace monitoring and control task. It is assumed that the invisible arc of the domestic stations is $197.25^{\circ}$ to $357^{\circ}$. Due to the complex composition of the BeiDou navigation system, it is difficult to distinguish multiple schemes in the same scene simulation. Therefore, they are separately by the link simulations of mission support programs for GEO satellites, IGSO satellites and MEO satellites in BeiDou navigation system.

1) Supported based on GEO Satellites

The GEO Satellites in Beidou navigation system are selected to support the space-based measurement and control system. The airspace monitoring and control tasks can be relayed through the navigation star G1 and G3. G1 is responsible for the arc of $290^{\circ}$ to $357^{\circ}$ as shown in Fig.1. G3 is responsible for the arc of $195.25^{\circ}$ to $290^{\circ}$ as shown in Fig. 2. The targets in other arcs are directly landed by the domestic stations. 
2) Supported based on IGSO Satellites

Select Beidou IGSO satellites to support the space-based measurement and control system for all airspace monitoring and control tasks. Two IGSO satellites or three IGSO satellites could be used to visualize the construction of the chains. As shown in Fig.3.

3) Supported based on MEO Satellites

The Beidou navigation system MEO satellites are selected to support the space-based measurement and control tasks. The multi-hop relay of MEO satellites can be used with the proper schedule. There are 24 MEO satellites in three orbits. The support schemes are diverse and complex, including the communication schemes of the satellites in same layer and multi layers. Here, only the satellites in the first orbital layer are taken as an example for support scheme. Link simulation is shown in Fig.4.

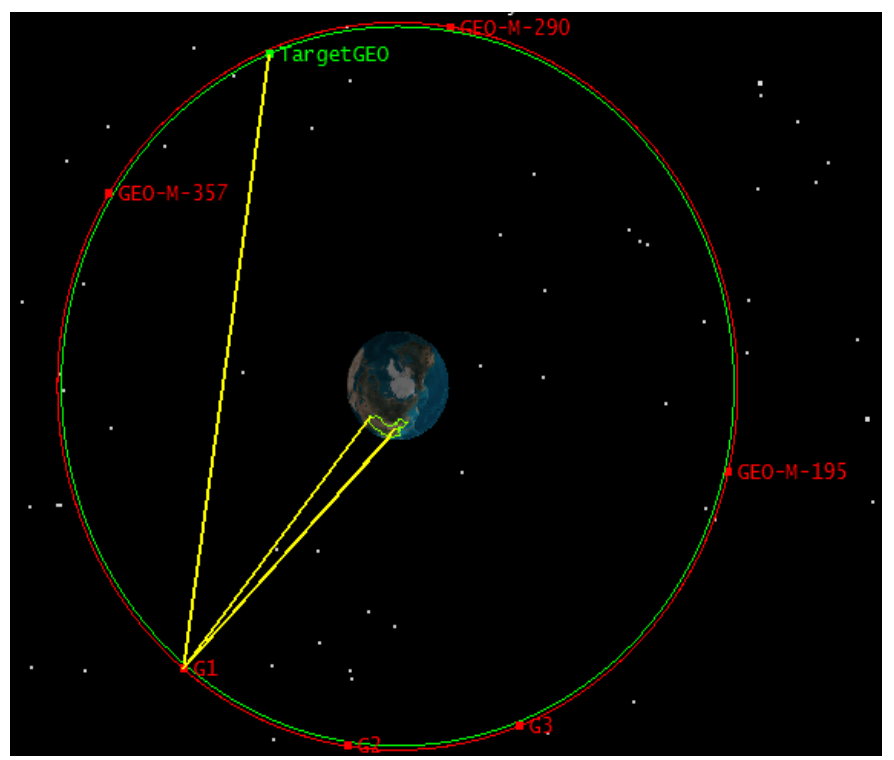

Fig. 1 The arc segment of G1 relaying (290 ${ }^{\circ}$ to $\left.357^{\circ}\right)$

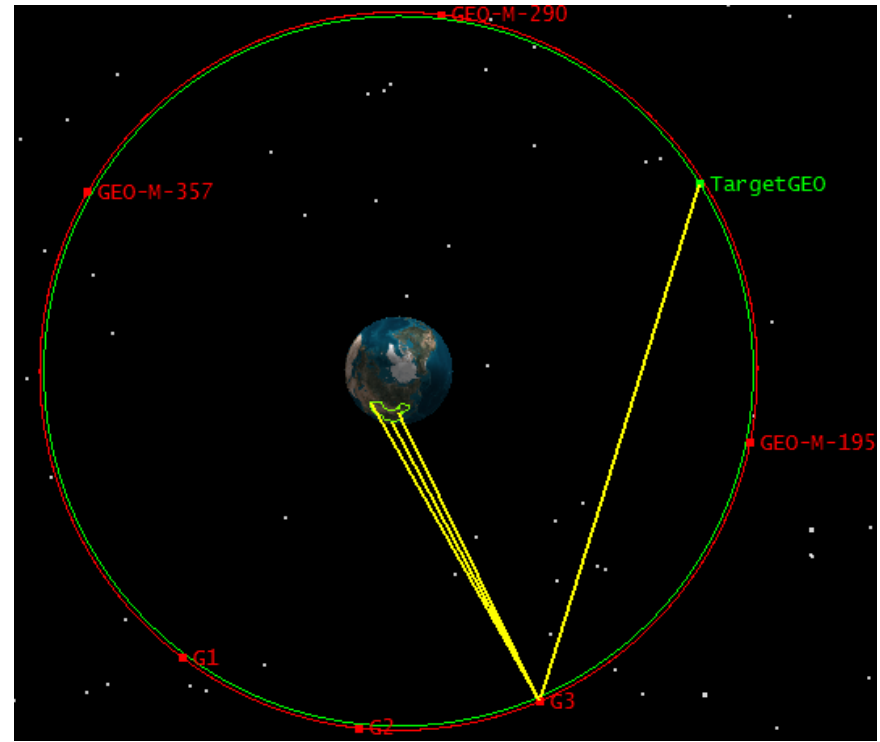

Fig.2 The arc segment of G1 relaying (195.25ํo 290) 


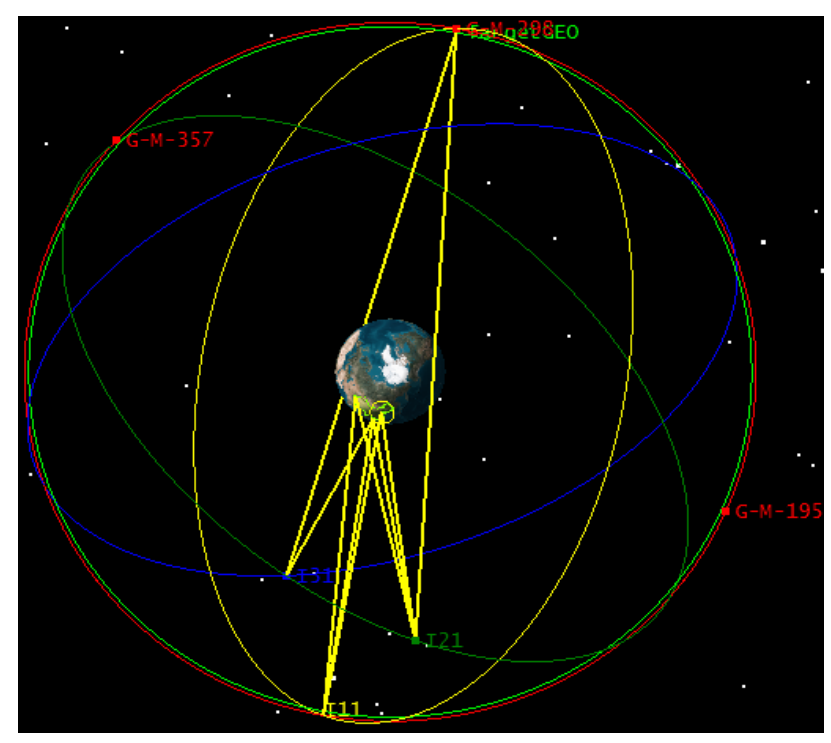

Fig. 3 Link simulation based on IGSO satellites

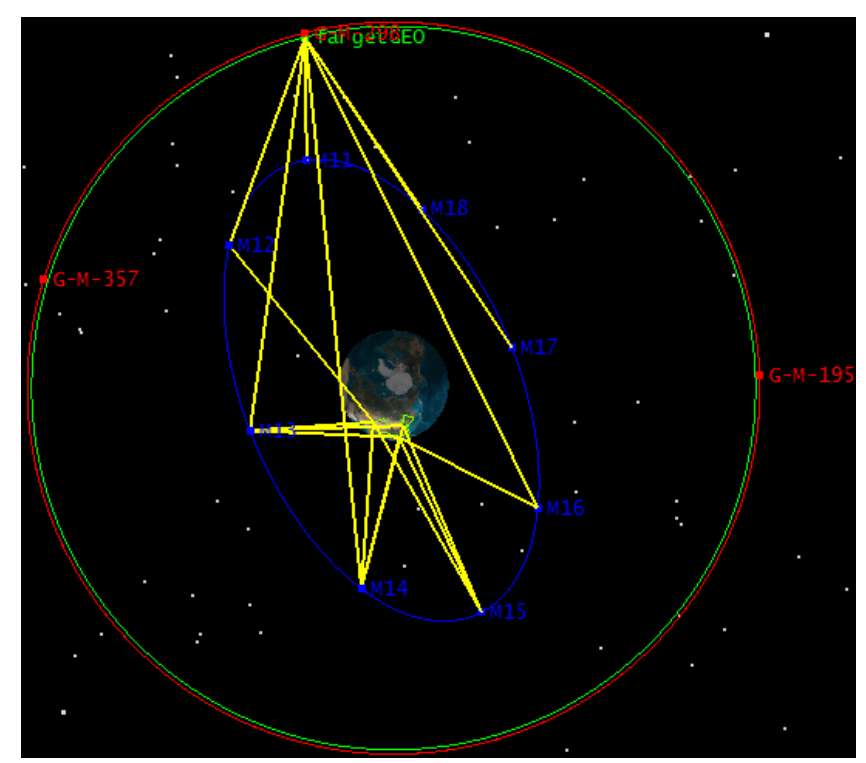

Fig. 4 Link simulation of MEO satellites in the first orbital layer

\subsection{Coverage simulation}

This paper analyzes the coverage of the support plans based on the requirements of monitoring and control tasks. If the scanning range of the satellites and ground stations is 60 degrees. Based on the link simulation, coverage analysis is performed.

The three GEO satellites (G1, G2, and G3) in the Beidou Global System are fixed at $80^{\circ}, 110.5^{\circ}$ and $140^{\circ}$ respectively, which are the same as geostationary orbit satellites in the traditional space-based measurement and control system. Because it is sheltered by the earth, G1 can not cover the orbital arc at $242.6^{\circ} \sim 278.7^{\circ}$, G2 can not cover the orbital arc at $273.1^{\circ} \sim 307.9^{\circ}$, and G3 can not cover the orbital arc at $302.6^{\circ} \sim 337.4^{\circ}$. The geometric visibility analysis is shown in Fig. 5. 


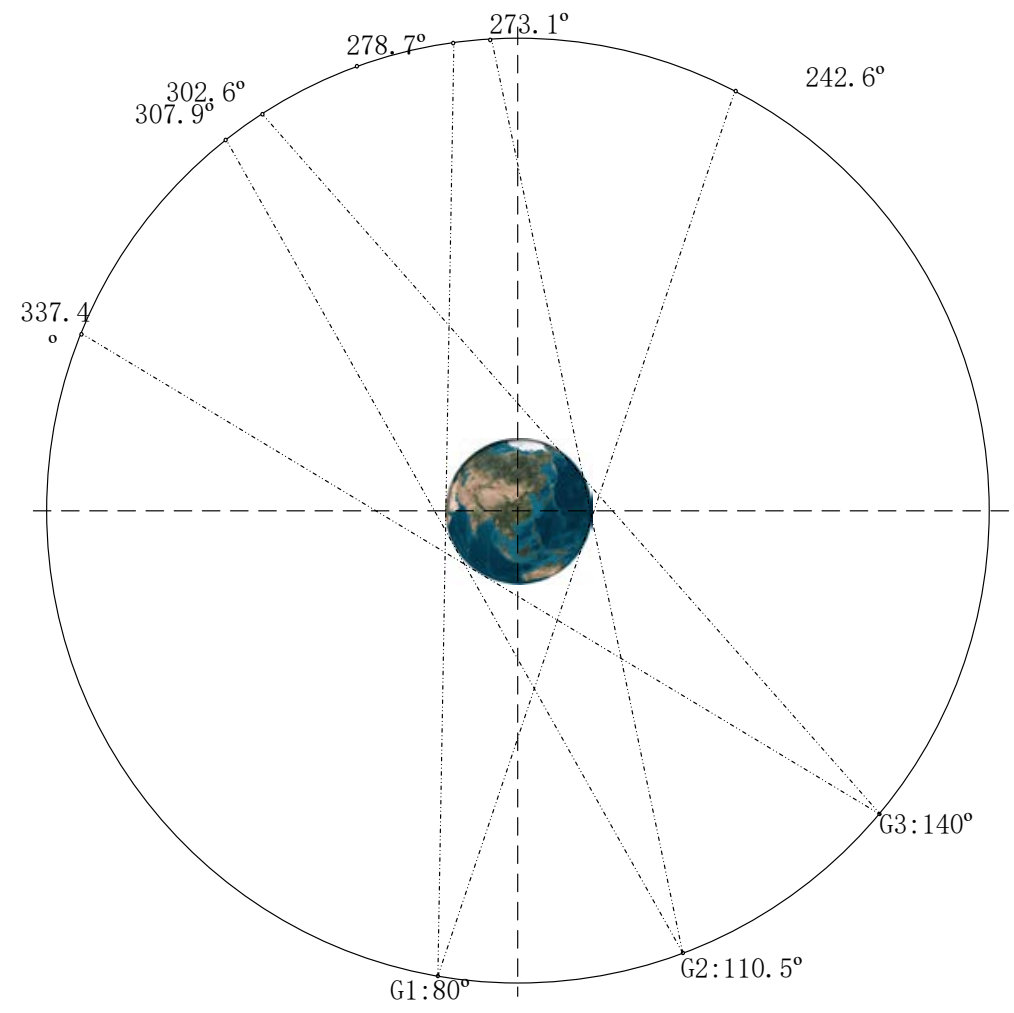

Fig.5 Analysis of uncoverable arcs of GEO satellites

Through the analysis, the support program of Beidou IGSO satellites selects any two IGSO satellites or three IGSO satellites. Utilize the switching of links to realize the full airspace monitoring and control tasks.

Based on the orbit and operational characteristics of IGSO satellites in the Beidou constellation, the arc at East $298^{\circ}$ is the most unfavorable to the observation. When the targets are at this arc, the visible relationships between IGSO satellites and targets are shown in Fig. 6.

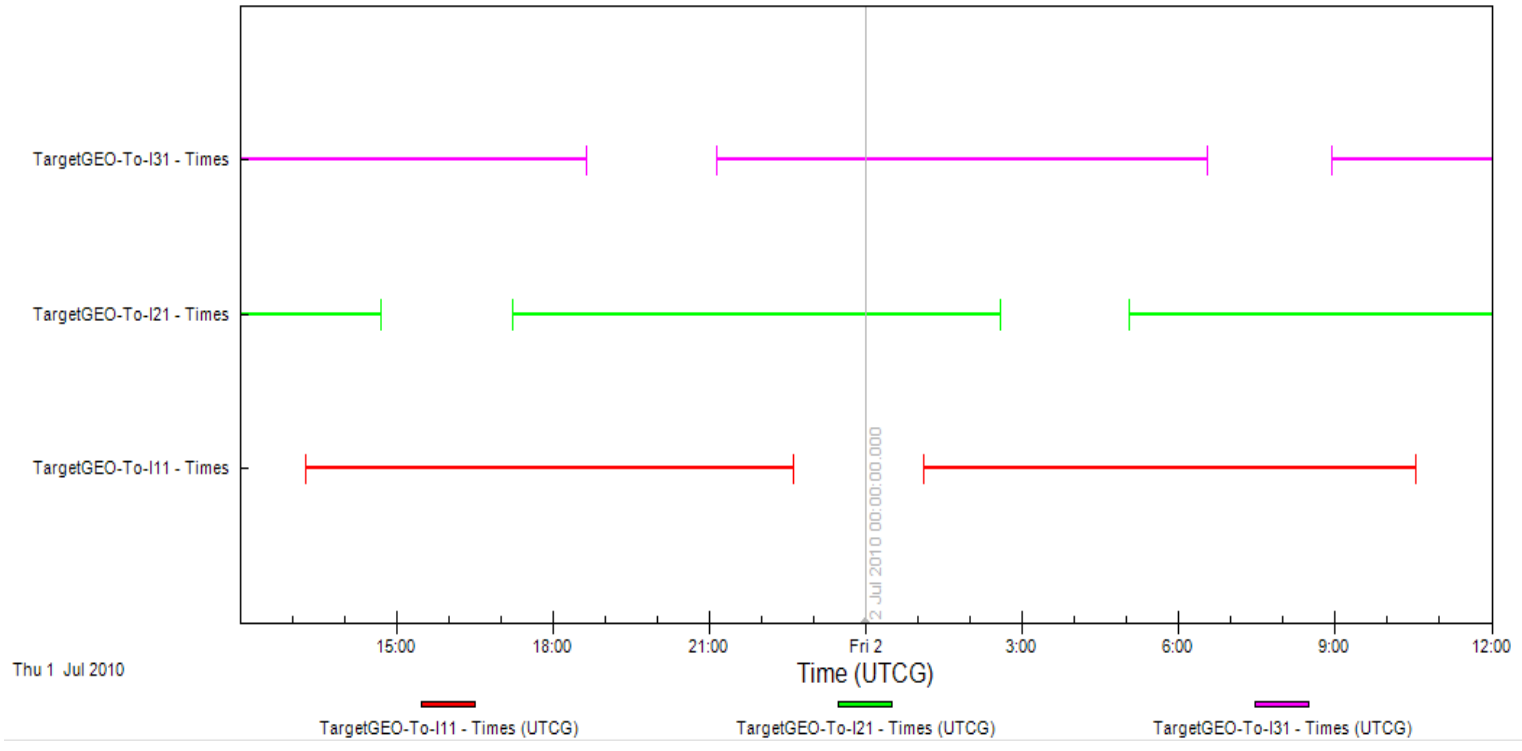

Fig. 6 Connectivity between IGSO satellites and targets

The coverage of MEO satellites should be used with the proper schedule for different applied scenes. 


\section{Conclusion}

Compared with coverage analysis for measurement and control link simulations, it is simple to establish inter-satellite communication links between targets and the Beidou GEO satellites, relatively complex with IGSO satellites, and most complex with MEO satellites. Simple topology is easier to maintain. Therefore, for the support of all airspace monitoring and control tasks, the GEO satellites support the best, followed by IGSO satellites, and finally MEO satellites.

\section{References}

[1] Li Yanhua, LU Manhong. Trends of Applications of Space-Based TT\&C System. Journal of Spacecraft TT\&C Technology. Vol. 31 (2008) No. 4, p. 1-4.

[2] Yang Hongjun. Latest Development Progress and Trends of Foreign Data Relay Satellite System. Telecommunication Engineering. Vol. 56 (2016) No. 1, p. 109-116.

[3] Zhang Qiang, Pei Yin, Wang Xiaohui, et al. Research on Vehicle's Telemetry Command and Communication System Based on TDRSS. Journal of Telemetry, Tracking and Command. Vol. 35 (2014) No. 1, p. 18-22 